\title{
Investigar En Ciencias Sociales: El Desafío Por El Análisis Científico
}

\section{Graciela Castro ${ }^{1}$}

Tipo de trabajo: Artículo

Material original autorizado para su primera publicación en el Journal de Ciencias Sociales de la Facultad de Ciencias Sociales de la Universidad de Palermo

\section{Recibido 28-9-2012 \\ Aceptado 23-10-2012}

\section{Resumen}

La función de la universidad se funda en tres ejes básicos: docencia, investigación y extensión. Todas ellas siempre han sentido la influencia del contexto histórico, social y político de cada tiempo. La investigación, en particular, ha sentido sobre sí los clivajes provenientes del contexto los cuales han influido en las prácticas de los actores universitarios. Junto a las políticas de estado es preciso detener la reflexión en lo que sucede en las ciencias sociales: sus problemas de investigación, los paradigmas teóricos y metodológicos y el impacto de las investigaciones en su relación con el mundo público y el académico. El presente ensayo es el resultado de prácticas investigativas de las cuales devienen reflexiones que procuran pensar la investigación como una tarea estrechamente vinculada con las demás funciones que competen a la vida académica incorporando la influencia de momentos y situaciones que provienen del contexto científico y político, en particular en Latinoamérica.

Palabras clave: investigación- ciencias sociales- formación- contexto

\section{Research in Social Science: the challenge for scientific analysis}

\section{Abstract}

The university's role is based on three basic axes: teaching, research, and "extension", all of which have always been influenced by the historical, social, and political contexts of the times. Research, in particular, has been affected by this fact, straining in turn all practices performed in the university. Along with state policies, it is necessary to stop reflecting on what is happening in Social Science: its research issues, its theoretical and methodology paradigms, and the impact it has in its relation with the public and the academic world. The present essay is the result of research practices that have provided insights on research as a highly related task with the other areas of academic life, taking into consideration -at the same time- the influence of moments and situations from the political and scientific context, especially in Latin America.

Keywords: research- social sciences- education- context

\footnotetext{
${ }^{1}$ Docente- investigadora en temas de ciencias sociales. FICES/Universidad Nacional de San Luis. Directora de Kairos, revista de temas sociales, UNSL. Directora de la Maestría "Sociedad e Instituciones" (UNSL). 


\section{Introducción}

Durante mucho tiempo, en particular en las ciencias sociales, se consideró que las actividades de docencia ocupaban de modo exclusivo el ámbito académico. Si alguno de los actores se sentía inclinado a preguntarse por alguna cuestión de su campo disciplinar debía recurrir a la literatura existente que, en general al tratarse de temas sociales, no siempre coincidía con el contexto sociocultural de su realidad inmediata, especialmente vinculado con las situaciones y hechos de Latinoamérica, dejando por consiguiente varias preguntas sin respuestas. Las tareas investigativas parecían estar destinadas a una elite privilegiada, al menos desde la perspectiva del imaginario social. Si bien en el nivel universitario las tareas de investigación incluían tanto las ciencias exactas como las sociales, en estas últimas parecía no percibirse una vinculación estrecha entre las actividades de docencia y las de investigación. Tal vez esa actitud estuviera atravesada con representaciones sociales que colocaban a la investigación y a los investigadores en planos diferentes al del docente. Sin embargo la vida académica requiriere una ligazón entre las tareas investigativas y las que corresponden a la docencia.

Cuando los fenómenos, hechos o actores sociales comienzan a inquietarnos más allá de lo habitual y nos plantean más preguntas que respuestas convencionales, ya estaríamos acercándonos al problema que nos interesa investigar. Estos reclaman de nuestra parte: curiosidad, creatividad, paciencia y rigurosidad en el tratamiento de los elementos e información. Aquí ya comenzamos a adentrarnos en cierta terminología propia de la jerga académica: ciencias, teorías, métodos, técnicas y dos aspectos esenciales que vinculan a todos aquellos conceptos. El primero y fundamental: el conocimiento, éste constituye el motor que orienta la búsqueda. Para acceder al conocimiento, el otro aspecto necesario, se vincula con la práctica de investigación. Ella nos conduce a interrogar, a observar cada aspecto del hecho o colectivo social que nos interese analizar. Los resultados de la investigación podrán plantear hipótesis que conduzcan a nuevas teorías y así al desarrollo científico.

Ahora bien, ¿cuál es la situación actual de las ciencias sociales y qué papel juegan los investigadores? ¿Continúa en el nuevo siglo la misma actitud hacia la ciencia y los objetos de investigación que existía en los años sesenta y setenta? Al respecto el sociólogo guatemalteco Edelberto Torres Rivas afirma que por aquellas décadas el conocimiento era una herramienta que se creía podía modificar la realidad y agrega "La historia demostró que la realidad era más terca, que no se cambia fácilmente y ese lazo se rompió" (2009; p. 65). En este nuevo contexto histórico el sociólogo guatemalteco considera que aparecen dos tipos de conocimiento: "el de las ideas y la cultura simbólica, por un lado, y el conocimiento instrumental por otro" (2009; pp. 65,66). A partir de este último tipo considera que se produce la transformación de académico-investigador en consultor. Esta condición del investigador no implica que se pierda el conocimiento, sino que el mismo tiene otra dinámica, sirve para otra cosa. $Y$ esta situación también coloca otro desafío a la actividad del investigador.

El presente ensayo procura reflexionar acerca de algunos aspectos vinculados con la investigación en las ciencias sociales, sus dificultades, la influencia de políticas de estado y su repercusión en las prácticas de los actores académicos. Así mismo se busca incorporar en el análisis la incidencia de paradigmas teórico- metodológicos como así también el impacto de los resultados 
de las investigaciones y el papel del investigador como ciudadano con sentido ético y mirada crítica frente al mundo social de su tiempo.

\section{La caja de herramientas}

Podríamos señalar que uno se acerca a la investigación científica cuando las preguntas superan a las certezas que otros proponen y los problemas sociales exhiben aspectos o características que requieren nuevas interpretaciones. Allí comienzan las búsquedas, la detenida observación del objeto de estudio, las indagaciones que aporten la necesaria información que nos conduzcan a análisis rigurosos y su consecuente interpretación científica. Indudablemente cuando hablamos de investigaciones no se puede soslayar la presencia humana. El premio nobel de química Prigogine, al hablar de la nueva alianza entre el hombre y la naturaleza, señalaba que no era posible comprender al investigador reducido a un espacio, casi como una torre de marfil. Por el contrario, debería considerarse que el investigador cuenta con su propia vida cotidiana la cual está atravesada por situaciones socioculturales al mismo tiempo que los propios fenómenos de estudio muestran su complejidad. Los estudios prigoginianos permiten comprender la transformación de los conceptos de la ciencia clásica y describir la metamorfosis de la ciencia en los siguientes términos: "El tiempo no es ya el centro de atención de los fenómenos inmutables... nos interesan las evoluciones, las crisis, las inestabilidades. Ya no queremos estudiar solamente lo que permanece, sino lo que se transforma" (Prigogine; Stengers; 1984; p.35).

En el último siglo el "conocer" había sido identificado con "saber manipular" y la psicología skinneriana es un ejemplo de esta interpretación ya que considera a los seres vivos como cajas negras: importan las "entradas" que son controladas, y las "salidas" que corresponden a las respuestas de los sujetos a la experimentación. De modo diferente a los enfoques de Skinner, la teoría prigoginiana está atravesada por la noción de movimiento: "Sabemos que no podemos siquiera garantizar la estabilidad del movimiento planetario" afirmaban Prigogine y Stengers, "y esta inestabilidad de las trayectorias son las bifurcaciones en donde volvemos a encontrar las fluctuaciones de nuestra actividad cerebral, que son hoy, nuestra fuente de inspiración" (1984; p. 298). ¿Alguien puede negar en estos tiempos que una de las características primordiales de la ciencia es precisamente contar con la noción de movimiento, de dinamismo? Quienes estudiamos los fenómenos sociales debemos enfrentarnos a problemas básicamente caracterizados por esa noción prigoginiana de movimiento y complejidad.

En la nueva alianza entre el hombre y la naturaleza, que se construye a partir de los principios de la termodinámica y el papel de la irreversibilidad para la comprensión de la flecha del tiempo, la construcción del conocimiento precisa de nuevas alianzas que incluyan las ciencias básicas, las ciencias humanas, las ciencias de la naturaleza y las ciencias sociales, pues el hombre es el producto de complejos procesos físico-químicos como también de la historia de su propio desarrollo, de la historia de su especie y de sus sociedades. La vinculación entre la ciencia y la cultura es esencial para la construcción del conocimiento en una etapa histórica que muestra profundos cambios científicos y tecnológicos. El cambio puede ser interpretado en el lenguaje del movimiento. Una de las posibles metáforas para la interpretación ha sido la del crecimiento orgánico, pero algunos cientistas consideran más apropiado recurrir a la metáfora del movimiento que pertenece a 
la dinámica de los sistemas complejos. Esta dinámica incluye el desarrollo embriológico, la ecología, la evolución biológica y los sistemas caóticos de la física posmoderna. Estas clases de sistemas tienen rasgos comunes: son sistemas dinámicos abiertos, que no presentan equilibrio y cuentan con la potencialidad de la autoorganización. Al respecto Lemke (1993) expresa que en estos sistemas los puntos centrales están en la comprensión de la trayectoria del desarrollo sobre el tiempo y en la interdependencia de los procesos y actividades que los integran

En las últimas décadas se ha desarrollado de modo intenso la tecnología la cual, junto a flujos económicos, culturales y políticos conformó lo que se denomina sociedad informacional o sociedad de la información. En ella el conocimiento deja de ser acumulativo, lo que demanda que el aprendizaje sea una actividad permanente y no una etapa de la vida. Esto significa que se debe tender a que los individuos sean capaces de acceder al conocimiento, seleccionarlo, analizarlo y luego desarrollar nuevos conocimientos.

Alcira Argumedo (1997) afirma que:

"Junto a otros cambios de magnitud, la Revolución Científico-técnica impone al conocimiento - que incluye información y capacidad innovativa- como el nuevo recurso estratégico que ha de definir el papel de los distintos países y regiones al comenzar el tercer milenio".

Y agrega más adelante que:

"El conocimiento emergente se define por su carácter flexible, transdisciplinario y con una consistente formación de base, que son las condiciones para garantizar eficiencia y creatividad en cada campo científico. Las Universidades deben promover un pensamiento crítico y riguroso capaz de articular diferentes conocimientos como modo de enriquecer y potenciar el conocimiento especializado" (Argumedo,http:// www.revistakairos.org/k01-07.htm).

Desde esta perspectiva, las Universidades deben generar nuevos modos de pensar, capaces de articular los conocimientos de las ciencias duras con los de las ciencias sociales y viceversa, no con la finalidad de actuar como una simple sumatoria de saberes, sino buscando nuevos abordajes que permitan plantearse nuevas respuestas con sentido creativo y crítico ante un tiempo histórico con problemáticas diversas.

Si se comprende que las actividades académicas precisan de la estrecha vinculación entre la docencia y la investigación y si esta última constituye un requisito a cumplir por los docentes, la propuesta de lograr una capacitación transdisciplinaria podría favorecer un acercamiento apropiado hacia las tareas investigativas, en tanto y en cuanto, ello permitiría no sólo definir problemas de estudio sino también la manera apropiada de realizar su tratamiento científico.

"El objeto de las ciencias sociales es histórico" afirma la investigadora brasileña María Cecilia de Souza Minayo (2009; p.33) y agrega: "Toda investigación social necesita registrar la historicidad humana, respetando la especificidad de la cultura que trae en sí y, de forma compleja, los trazos de acontecimientos de corta, media y larga duración, expresados en sus bienes materiales y simbólicos" (2009, p. 33). He aquí una cuestión esencial en las investigaciones del área social: la relación entre el 
investigador y el objeto de investigación. Ambos comparten la naturaleza histórica y es precisamente la historia el centro de la vida cotidiana, entendida ésta como el espacio de interacción social por excelencia en el cual el sujeto construye la identidad social y la subjetividad. Este espacio es común entonces al investigador y su objeto de estudio. La vida cotidiana, por consiguiente, se objetiva en las instituciones y la urdimbre de significaciones que en ellas se construye. Los problemas sociales que concitan el interés de los investigadores sociales se relacionan con los colectivos sociales: sus conflictos, sus luchas, sus rebeliones; en definitiva, la vida en sociedad y las complejas relaciones interpersonales.

A diferencia de quienes estudian elementos de la química, la física u otras disciplinas de las ciencias exactas, en las ciencias sociales el investigador puede convivir con su objeto de estudio, de modo amoroso o aversivo pues coinciden en un espacio histórico que plantea un desafío importante en tanto y en cuanto se pone en juego la cosmovisión del investigador, la ideología, la responsabilidad social y la necesaria formación teórica. A este desafío puede agregársele otro relacionado con el abordaje metodológico que elija el investigador para sus tareas investigativas. La recurrencia a metodologías cualitativas a contrariu sensu de algunas creencias requiere de una rigurosa formación teórica. Este abordaje puede demandar la utilización de análisis de contenido, del discurso o un análisis hermenéutico-dialéctico. En síntesis y, retomando las afirmaciones de la socióloga brasileña De Souza Minayo "El universo de las investigaciones cualitativas es el cotidiano y las experiencias del sentido común, interpretadas y reinterpretadas por los sujetos que las vivencian" (2009, p. 21). Acá se advierte claramente el papel de la palabra y sus significados los cuales son construidos por los grupos, interpretados, reinterpretados, como señala la investigadora, por quienes vivencian los diversos aspectos que se construyen en la vida cotidiana de cada sujeto y que se manifiestan - como expresa Agnes Héller (1987, p. 227)- en las objetivaciones, entendidas éstas como los sistemas de referencia que el hombre debe apropiárselos. En ese sentido la investigadora húngara determina tres instancias de las objetivaciones: las genéricas en sí que constituyen el fundamento necesario para el crecimiento del hombre, comprendiendo a los utensilios, productos, usos y lenguaje; las objetivaciones genéricas para-sí constituyen la encarnación de la libertad humana; y las objetivaciones en sí y el para-sí comprenden a la ciencia, las religiones, las estructuras políticas entre otras. El investigador social debe detenerse en el estudio y análisis de esas expresiones de la vida cotidiana de los sujetos que son atravesadas por aquellas objetivaciones y las significaciones que de ella surgen. Pero al mismo tiempo, el investigador social también integra ese mundo social donde construye su propia vida cotidiana, con las significaciones que cada uno establece en función de su historia y posibilidades. Esta situación también constituye un desafío que los investigadores deben enfrentar para el estudio de los hechos sociales.

\section{Consideración del contexto}

Ruth Sautu (2003) en su texto Todo es teoría afirma que "El primer paso para la definición del objetivo de la investigación es discutir el contexto socio-histórico en el cual suponemos que el proceso, fenómeno o suceso tiene lugar"; y agrega que las preguntas son

¿De qué se trata?, ¿quiénes suponemos que están involucrados?, ¿cuál es 
la naturaleza de esos quienes son: entidades reales, comunidades, etc?, ¿cuál es la situación, lugar o entorno en que tiene lugar o dónde están localizadas las entidades que suponemos están implicados?. (Sautu, 2003; p.13)

¿Qué características identifican a la sociedad contemporánea? Los elementos que dan cuenta de tales aspectos remiten a la interdependencia de los mercados; y cambios sociales y culturales que se definen por una deslegitimación de las instituciones y organizaciones sociales. El segundo aspecto se refiere al papel del conocimiento como recurso estratégico para el desarrollo. Todo ello permite identificar a la sociedad contemporánea como global e informacional. Es global porque la producción, el consumo, la circulación y los componentes del proceso productivo están organizados en una red de vínculos entre los agentes económicos. Es informacional porque la productividad y la competitividad de los agentes económicos dependen de la capacidad para generar, procesar y aplicar la información basada en el conocimiento. La relación entre ambos conceptos está dada en la noción de red. La sociedad red se caracteriza por la globalización de la economía, la cultura de la virtualidad y la transformación de la concepción del espacio y el tiempo (Castells, 1997)

La sociedad de la información, afirma Raúl Trejo Delarbre se basa en el conocimiento “ (...) y en los esfuerzos por convertir la información en el conocimiento. Cuanto mayor es la cantidad de información generada por una sociedad, mayor es la necesidad de convertirla en conocimiento"(1999; p. 11). La información tiene un valor social y económico que proviene del reconocimiento individual o colectivo y de la necesidad que manifieste la sociedad por utilizarla. La sociedad y los gobiernos reconocen el valor de la información cuando admiten que el poder está vinculado a ella. Pero la riqueza de la información puede perderse si no se difunde, de allí que la lectura es el complemento necesario. La sociedad de la información no sólo considera la disposición, el acceso y el uso de la información, sino que también tienen en cuenta la necesidad de estimular la producción informativa en los múltiples grupos sociales que forman la diversidad social.

Los países latinoamericanos durante las décadas de 1960 y 1970 soportaron terribles dictaduras que no sólo afectaron sus sistemas de gobierno, cercenando los derechos constitucionales, avasallando los derechos humanos, sino también destruyeron el desarrollo del sistema científicotecnológico que -en particular en Argentina- venía desarrollándose de modo importante. Para las ciencias sociales, especialmente, las dictaduras significaron una etapa de involución, de destrucción que condujo a miles de investigadores al exilio y enmudecieron a los que no pudieron irse del país. El miedo y el terrorismo de estado, produjo una desestructuración de la vida cotidiana también de los cientistas sociales, obturando la posibilidad de estudiar y analizar el comportamiento de los fenómenos sociales atravesados por un tiempo histórico de violencia y destrucción. Tras la reapertura democrática, durante la década de 1980, se hizo preciso reconstruir el sistema científico en el país pues era necesario tener respuestas científicas que ayudaran a entender los clivajes ocurridos durante la dictadura. Con posterioridad, durante la década de 1990, las consecuencias sociales del neoliberalismo pusieron al descubierto otros problemas que demandaron su estudio profundo con criterios científicos.

Sin lugar a dudas los cambios socioeconómicos desestructuraron la vida cotidiana de los ciudadanos: la aplicación de políticas neoliberales - las cuales quedaron expresamente señaladas en el Consenso de Washington- que propiciaban desregulación laboral y privatizaciones de organismos 
hasta entonces públicos (Guiñazú, 2002), trajeron como consecuencia altos índices de desocupación que ocasionaron graves crisis sociales. Alguien podría preguntarse qué vinculación tiene la crisis social con la tarea investigativa. Dos vías de análisis se presentan ante este interrogante: el que corresponde a los actores sociales, su inserción laboral y las condiciones del mismo; y los temas que emergen ante la crisis. Así como no se pone en discusión que uno de los temas centrales durante la dictadura fue el miedo atravesando la cotidianidad de los ciudadanos, durante los noventa la precarización laboral y la desocupación consecuente colocaron la incertidumbre social y el desencanto político como ejes de debate.

Junto a los problemas y desajustes planteados por la aplicación de políticas neoliberales hay que tener en cuenta los cambios culturales: desterritorialización de las comunicaciones que superaron las fronteras geográficas y afectaron las culturas regionales al mismo tiempo que plantearon la necesidad de respetar la diversidad cultural. Junto a estas situaciones el consumo se estableció como un elemento de suma importancia en la construcción de la subjetividad. Las consecuencias de la crisis traspasaron las puertas de las escuelas, institutos y universidades: cada alumno y cada docente agregaron a sus mochilas dificultades provenientes del contexto social. En consecuencia también los problemas a investigar se presentan atravesados por las características del contexto social.

El nuevo siglo ha mostrado un escenario particular no solo a nivel mundial sino también en Latinoamérica. Si pensamos en la noción de desenclave planteada por Giddens (1994) como una manera de comprender la desconexión entre los hechos, el tiempo y el espacio, el mundo se volvió global aunque los beneficios no sean extensivos para todos los países. Allí está Haití mostrando su lacerante pobreza agudizada tras el terremoto en 2010 y más próximo en el tiempo, similares situaciones vividas en Chile, si bien terribles y angustiantes como en Haití pero con potencialidades diferentes para enfrentar la crisis, sin que por ello se desconozca las diferencias sociales hacia el interior de la sociedad chilena. Pero la realidad latinoamericana no sólo está atravesada por movimientos telúricos; las situaciones políticas, sociales y económicas han construido escenarios donde el desencanto político conspira contra la construcción de una ciudadanía social activa pero al mismo tiempo emergen movimientos que dan protagonismo a colectivos sociales hasta entonces marginados, como los pueblos originarios, las minorías sociales, de género, entre otros. Junto a esto también se asoman otras paradojas: avances tecnológicos junto a luchas salariales de los trabajadores y deserciones educativas. En 1999, según el estudio de la Consultora Equis, era altamente preocupante el elevado porcentaje de jóvenes entre 15 y 24 años que no trabajaba, ni buscaba trabajo, ni estudiaba. Y más allá de las cifras reales o ficticias del INDEC la pobreza convive con los barrios cerrados. Este es el escenario de Latinoamérica y en el cual el investigador está inmerso junto a sus objetos de investigación.

\section{La investigación como política de estado}

En la elección de los problemas a investigar el cientista no puede soslayar el contexto social y político en el que está inmerso pues, además, el impacto de los resultados de sus investigaciones nunca será totalmente neutral. Los países del tercer mundo, emergentes o en vías de desarrollo, de acuerdo a la denominación a la cual cada uno prefiera, presentan determinadas características 
socioeconómicas y políticas que demandan contar con una política científica particular. Un aspecto de sumo interés es considerar la necesidad de integración entre los países, atravesada no sólo por aspectos económicos sino también sociales y culturales y la ciencia es un elemento fundamental para lograr el desarrollo y la integración. Al respecto Aréchiga, investigador mexicano, asevera que

La investigación científica es una tarea colectiva, basada en el intercambio libre de ideas, enriquecida por la colaboración y la complementariedad y cuyos frutos están en principio disponibles para todos. Sin embargo, para que la ciencia y la innovación tecnológica constituyan elementos de cambio y de mejoramiento de las condiciones sociales y económicas en los países de la región, se requieren de cambios fundamentales en las políticas internas y relaciones multi- y bi-laterales. (Aréchica,1998; p. 19).

El nuevo siglo ha ido presentando importantes cambios políticos, económicos y culturales en los países de Latinoamérica: tras padecer durante los '90 el impacto de las políticas neoliberales que dejaron graves secuelas sociales, los primeros años del siglo XXI mostraron cambios ideológicos en la conducción de varios países. Si bien cada uno de ellos exhibe sus perfiles propios, no ocultan sus parecidos de familia, en particular en las políticas públicas y en la necesidad de construir bloques y foros internacionales que favorezcan la integración regional. Allí se puede ubicar al MERCOSUR, la UNASUR y la CELAC sólo por nombrar algunos de tales organismos regionales. Es innegable el protagonismo que estos foros han tenido en la última década -desde el punto de vista político fundamentalmente- que ha permitido integrar a la región, sin embargo no se visualiza aún una integración cultural que favorezca el desarrollo científico-tecnológico entre los países latinoamericanos.

Junto a la necesidad de integración entre los países es preciso considerar el papel que les compete a cada uno de los Estados en su vinculación con la ciencia. En la Conferencia Científica de la VI Cumbre Iberoamericana de Jefes de Estado y de Gobierno, realizada en Santiago de Chile en 1996, se acordó, entre otras cuestiones que

Laciencia y la tecnología contribuyen significativamente a elevar la productividad y competitividad de los países, pero su aporte al desarrollo es mucho más amplio, contribuyendo a elevar la calidad de vida, solucionar problemas del medio ambiente y aumentar la eficiencia de la administración del Estado,

señalando a continuación que

Corresponde al Estado asumir un papel activo en la fijación de grandes objetivos y prioridades, asignación específica de recursos públicos de la inversión privada en ciencia y tecnología; así como, en la implementación de mecanismos e instrumentos de política científica y tecnológica. En otras palabras, políticas públicas estables y coherentes (http://www.oei.es/vicumbre.htm). 
En diciembre de 2007, por decisión de la presidenta Cristina Fernández de Kirchner se creó en Argentina el Ministerio de Ciencia y Tecnología cuya finalidad es "orientar la ciencia, la tecnología y la innovación al fortalecimiento de un nuevo modelo productivo que genere mayor inclusión social y mejore la competitividad de la economía argentina, bajo el paradigma del conocimiento como eje del desarrollo" (http://www.mincyt.gov.ar/ministerio/presentacion/index.phpecono).

En 1996, se creó la Agencia Nacional de Promoción Científica y Tecnológica, como parte de una reforma del Sistema de Ciencia y Tecnología. En la actualidad depende administrativamente del Ministerio de Ciencia, Tecnología e Innovación Productiva.

La Agencia, a través de sus dos Fondos (FONCyT y FONTAR), financia el desarrollo de investigaciones científicas, innovaciones y modernizaciones tecnológicas de empresas, científicos e institutos de investigación de todo el país. Administra al FONSOFT, fondo fiduciario creado a partir de la sanción de la Ley de Promoción de la Industria del Software, y también al FONARSEC, cuyo objetivo es desarrollar capacidades críticas en áreas de alto impacto potencial. (http://www. agencia.gov.ar/spip.php?article21)

Las universidades públicas argentinas basan sus actividades en tres funciones, fundamentalmente: docencia, investigación y extensión. Dos organismos creados durante la década del '90 - en jurisdicción del Ministerio de Educación de la Nación- tuvieron una fuerte influencia en el ámbito universitario, tanto desde el aspecto institucional como en las propias prácticas de sus actores. El primero de ellos fue la conformación del Programa de Incentivo a Docentes- Investigadores y está "destinado a la promoción de la investigación en el ámbito académico, fomentando una mayor dedicación a la actividad universitaria y la creación de grupos de investigación" (http://www.me.gov. ar/spu). El otro fue CONEAU (Comisión Nacional de Evaluación y Acreditación Universitaria) entre cuyas funciones se advierten las siguientes funciones:

La evaluación externa de las instituciones universitarias; la acreditación de carreras de posgrado y carreras de grado reguladas por el Estado; y la emisión de recomendaciones sobre los proyectos institucionales de nuevas universidades estatales y de las solicitudes de autorización provisoria y definitiva de establecimientos universitarios privados (http://www.coneau.edu.ar/coneau/index.html\#).

La implementación de ambos programas institucionales no estuvo exenta de dificultades que provocaron resistencias y apoyos por parte de los actores universitarios, en tanto y en cuanto, la aplicación de las políticas surgidas de aquellos organismos tuvo una decisiva incidencia en las prácticas universitarias. Los discursos de quienes se oponían a la implementación de las políticas surgidas de ambos programas colocaban su basamento en aspectos ideológicos fundamentalmente. Tal actitud no resultaba caprichosa por cuanto durante la década del '90 las políticas surgidas desde el gobierno nacional se caracterizaron por su impronta neoliberal y las que correspondieron al área educativa también estuvieron atravesadas por el mismo signo ideológico. Tanto el Programa de Incentivo a Docentes- Investigadores como en CONEAU contaban con apoyo financiero del 
Banco Mundial, de allí una de las mayores resistencias por parte de quienes entendían que la implementación de ambas políticas introducían la mercantilización y desconexión con la realidad social, cultural y política del país. Un mecanismo común a ambos programas es la evaluación; uno de ellos destinado a investigadores y el otro a instituciones y carreras.

En lo que corresponde al Programa de Incentivos es interesante detenerse en aspectos que muestran la modificación en la cantidad de docentes- investigadores que se incorporaron en las diversas universidades argentinas al programa, según datos aportados por la Secretaría de Políticas Universitarias del Ministerio de Educación de la Nación:

\begin{tabular}{|l|l|l|l|l|l|}
\hline & 1998 & $\mathbf{2 0 0 0}$ & $\mathbf{2 0 0 2}$ & $\mathbf{2 0 0 4}$ & $\mathbf{2 0 0 6}$ \\
\hline $\begin{array}{l}\text { Investigadores } \\
\text { incentivados }\end{array}$ & 16.905 & 18.704 & 18.142 & 16.545 & 19.899 \\
\hline $\begin{array}{l}\text { Total de } \\
\text { investigadores en } \\
\text { el programa }\end{array}$ & 26.645 & 30.040 & 26.460 & 28.814 & 34.854 \\
\hline $\begin{array}{l}\text { Proyectos } \\
\text { incentivados }\end{array}$ & 5.367 & 5.363 & 5.280 & 5.552 & 6.587 \\
\hline
\end{tabular}

Fuente: http://www.me.gov.ar/spu/guia_tematica/incentivos/incentivo.html

Del cuadro anterior se percibe que en el año 1998, tras realizarse la primera convocatoria nacional del Programa de Incentivo, se incorporó 26.645 docentes- investigadores en las universidades argentinas; mientras que en 2006 la cantidad había ascendido a 34.854. Los datos estadísticos muestran sin dudas un crecimiento importante en las cifras. Sumado a esta información y desde un análisis cualitativo, los funcionarios de las respectivas instancias de Ciencia y Técnica de las universidades como así también quienes integran el Banco de Evaluadores -conformado tras la implementación del Programa para actuar como pares evaluadores- han podido observar desde el inicio del Programa hasta la actualidad no sólo un aumento en la cantidad de investigadores que se incorporaron a dicho programa, sino fundamentalmente un crecimiento y rigurosidad en la productividad científica de los investigadores junto al avance del perfeccionamiento académico a nivel de posgrado. Ambas vías de información permiten ciertas lecturas vinculadas con la investigación y la formación de los universitarios: una de ellas relacionada con situaciones económicas, pues tras la implementación de los programas institucionales ya mencionados, los docentes-investigadores incorporados al programa de incentivo comenzaron a recibir en sus salarios un monto extra - de modo semestral y que no siempre coincide con el ciclo académico vigente- de acuerdo a la categoría obtenida en dicho programa que comprende desde la inicial identificada como $\mathrm{V}$ hasta la de quienes integran el Banco de evaluadores: I y II. En cuanto a la formación de posgrado, y durante los últimos años, quienes cuentan con carreras de doctorados y maestrías finalizadas, también reciben en su salario montos que se suman al mismo. Si bien tal monto no resulta altamente significativo en términos económicos para la mayoría de los investigadores, constituye un aspecto motivador interesante. Ahora bien, desde el punto de vista estrictamente relacionado con las prácticas investigativas -dado que el programa de incentivo plantea requisitos académicos y características que deben tener 
en cuenta los investigadores en sus funciones y en la productividad- le fue otorgando a la tarea rigurosidad y parámetros científicos que mostraran los avances en las prácticas y que posibilitan que las mismas sean evaluadas con criterios comunes en todas las universidades por parte de los pares evaluadores.

Desde el inicio del Programa de incentivos como así también de CONEAU, a través de las instancias de gobierno que corresponden, se ha ido efectuando ajustes en algunos elementos de su implementación. Sin dudas -estas políticas y sus instrumentos de evaluación- siempre deberían ser pasibles de modificaciones a partir de las situaciones que muestran las instituciones educativas y los condicionantes culturales y políticos por las que atraviese en país.

\section{¿Qué hacer con el producto de la investigación?}

Una expresión común en el ámbito científico es aquella que expresa que "la ciencia que no se difunde no existe". El proceso investigativo requiere que sus resultados sean comunicados a la comunidad científica y también divulgado a la sociedad en general. Ahora bien, un elemento central en la tarea de difusión es la palabra. Si bien en toda relación humana la recurrencia a la palabra es esencial para permitir el proceso comunicacional interpersonal, en la constitución de las ciencias sociales resulta fundamental apelar a la palabra, ella se transforma en la vía regia para objetivar los pensamientos, las ideas que surgen en consecuencia, las búsquedas que orientarán las investigaciones y el medio que permitirá compartir los resultados a que arribe el investigador. De allí que para los cientistas sociales la palabra deviene central en sus prácticas. Ella halla en la lengua sus ropajes apropiados para establecer la comunicación entre los grupos. A diferencia de otros artefactos creados por la sociedad, la palabra no establece límites per se; el modo en que la construye el hombre puede favorecer la belleza, la muerte o la creación. Como afirma Ivonne Bordelois (2003; 70): "La derrota de la palabra implica una ceguera letal, un leso crimen de humanidad, un craso fracaso que necesitamos conjurar por todos los medios a nuestro alcance para no descender al infierno que nos proponen nuestros enemigos". El cuidado por la palabra no resulta de un banal cuidado por aspectos estéticos o meramente literarios, sino por el contrario, establecer con ella un vínculo amoroso y responsable, como afirma la propia Bordelois "nos singulariza como individuos" (2003; p. 22) lo cual no es un detalle menor y carente de importancia pues la singularización puede asimilarse a lograr la autonomía de la vida cotidiana (Guattari; Rolnik 2006). Este proceso deviene esencial para advertir y desarrollar dispositivos que permitan enfrentar el disciplinamiento y control que exhiben las instituciones dominantes.

Para los cientistas sociales la recurrencia a la palabra es fundamental, de allí la necesidad que ya en etapas de formación de los investigadores no se descuide un constante crecimiento y desarrollo en los discursos científicos. Para ello se requiere una formación integral de los cientistas que supere los espacios propios de su área disciplinar y una práctica recurrente de la lectura aún de textos literarios. En tiempos donde muchos tienden a bastardear la palabra, no es un detalle menor que los investigadores, y en particular los de ciencias sociales, coloquemos a la palabra con el respeto y cuidado que ella merece entre nuestras prácticas. 
En el siglo XXI las personas pueden utilizar la información

"sin necesidad de estar asociado a la academia, la investigación o una biblioteca. Pasa rápidamente, de la consulta de un diario impreso, a analizar la información de una página Web o a comentar, vía Internet, con un colega lejano, los movimientos de la bolsa." (Morales Campos, 2001, http://www.revista.unam.mx/vol.2/num2/art1/ index.html)

Cuando se habla de la información y el poder que implica su posesión no se puede dejar de hacer referencia, aunque sea de modo breve, al papel que corresponde a los intelectuales quienes, se supone, tienen como capital esencial la posibilidad de contar con información vinculada con los fenómenos sociales. De allí que la ética es un aspecto fundamental en ese análisis. Al respecto Michel Foucault afirmaba que

El papel del intelectual no consiste en decir a los demás lo que hay que hacer. ¿Con qué derecho podría hacer esto? El trabajo de un intelectual no consiste en modelar la voluntad política de los demás, estriba más bien en cuestionar, a través de los análisis que lleva a cabo en terrenos que le son propios, las evidencias y los postulados, en sacudir los hábitos, las formas de actuar y de pensar, en disipar las familiaridades admitidas, en retomar la medida de las reglas y de las instituciones y a partir de esta re-problematización (en la que desarrolla su oficio específico de intelectual) participar en la formación de una voluntad política (en la que tiene la posibilidad de desempeñar su papel de ciudadano) (Foucault, 1985; p. 10)

La difusión de los resultados de una investigación no sólo pone en cuestión el papel del intelectual como ciudadano sino que hay otro aspecto que provoca más de un conflicto: la exposición del intelectual a través de la difusión de los resultados de sus trabajos lo coloca ante la posibilidad de evaluaciones y críticas por parte de sus pares. La evaluación es un proceso que, desde mi opinión, merece un sinceramiento y debate sin prejuicios. Mis colegas psicólogos acertadamente, creo, expresarían que en este tema se pone en juego una profunda herida narcisista. Pero la evaluación requiere de dos actores: el evaluador y el evaluado. Retomando el análisis foucaultiano podríamos entender esa asimétrica relación a partir de la microfísica del poder donde el disciplinamiento penetra los cuerpos y graba la norma en la conciencia. Es indudable, y no podemos ocultarlo, que si se coincide al entender que ningún potencial científico es neutro en sus consecuencias, tampoco está exento el ámbito académico del juego de poderes que se pone en acción frente a las actividades vinculadas con la investigación, ya no en el plano científico sino propio de las relaciones interpersonales. Desde Galileo hasta acá sobrarían los ejemplos para pensar en aquellas relaciones. Pero la ciencia no puede quedar reducida a las relaciones personales. El estudio de los fenómenos sociales supera esas relaciones y por consiguiente sus resultados y consecuencias requieren ser compartidas y difundidas con la comunidad científica. Y es acá donde se expone el investigador, por ello deberíamos considerar que se puede coincidir o no con las evaluaciones pero ellas forman parte de aquello que en términos de Bourdieu diríamos son las reglas del juego. La soberbia puede 
convertirse en nuestro peor enemigo si no advertimos que el proceso investigativo es una diaria construcción que deviene de una constante formación y un diálogo permanente entre pares. El problema de nuestra investigación debería ser el tema central: agudizar los sentidos para escuchar y sentir sus expresiones y luego poner en acción la inteligencia.

Otro elemento de suma importancia en las prácticas investigativas es la constante formación que debemos realizar los investigadores. Frente a la complejidad de los fenómenos sociales siempre resulta preciso y apropiado contar con las necesarias herramientas teóricas que nos permitan un abordaje certero de los problemas en estudio. Sabemos que ningún fenómeno puede ser analizado desde la perspectiva de una sola área disciplinar; es preciso que los investigadores, junto a la especialización que cada uno puede haber logrado, se apropien de paradigmas y herramientas teóricas de otras disciplinas. Ello demanda una constante capacitación en la cual debe incorporarse una formación integral que incluya aspectos políticos, culturales, sociales y económicos.

\section{A modo de síntesis}

Aunque pueda resultar algo sin sentido, vale la pena decírnoslo una vez más: la investigación científica no está restringida a elegidos, a aquellos que pueden autodenominarse pomposamente expertos. Considero que es una actividad que requiere un constante aprendizaje y una dosis de humildad como para percibir nuestros errores y nuestros límites.

Tal vez, no resulta azaroso denominar ciertas acciones como propias de la "cocina de la investigación". Buscando analogías para el final hallaríamos objetos, fenómenos sociales, utensilios, técnicas, teorías y la puesta en marcha de algo que comienza de modo artesanal, casi jugando, observando, probando hasta lograr el producto apropiado. En todo ese proceso no puede estar ausente la creatividad, la perseverancia, aguzando los sentidos hasta sentir la propia voz de nuestro objeto de estudio. Sin duda no es una tarea para un día y a realizar en un solo día. Tampoco las comidas se preparan en un instante y una sola vez. Debería ser una elección que no concluya, en lo académico, tratando de responder a requerimientos institucionales. En nuestra analogía de ámbitos, algunos pueden transcurrir toda su vida viendo a la cocina como un mero lugar de tránsito; otros detendrán sus pasos para probarse a sí mismos si son capaces de elaborar deliciosos platos; algunos se nombrarán investigadores como una credencial meritocrática mientras otros buscarán respuestas que les permitan comprender a los fenómenos sociales sumando indagaciones, observaciones, dejando hablar sin tiempo al objeto, buscando respuestas que nos permitan comprender los fenómenos sociales que estudiamos, y al final, compartir enfoques con colegas y tras ello volver una y otra vez con los análisis.

Al mismo tiempo tendríamos que darnos cuenta que es posible poner el placer en escena: así como en la creación de las comidas también la investigación pone en juego las energías, la paciencia, las búsquedas, los temores, la soledad, el esfuerzo y la satisfacción de entender las razones del comportamiento de nuestro problema de estudio.

Junto a la necesidad de difundir los avances de las investigaciones - para lo cual devienen importantes las reuniones académicas y debates con colegas- como así también las publicaciones con rigor científico, es indudable que desde las instancias que corresponden a los gobiernos nacional, provincial y/o municipal se convoquen y consideren las tareas investigativas que se efectúan en 
el marco de las universidades, en particular las que corresponden a las ciencias sociales. Desde estas áreas disciplinares es mucho lo que podría aportarse para el conocimiento de los fenómenos sociales y su vinculación con la implementación de las política sociales.

En los últimos años desde las políticas estatales y desde el discurso oficial se viene colocando en un plano de protagonismo a las ciencias exactas y los investigadores de la misma área disciplinar y tecnológica; se menciona con asiduidad el tema de la repatriación de investigadores. No es posible desconocer ni ignorar que desde el estado se ha colocado en un lugar de importancia al desarrollo científico y tecnológico. Ahora bien, la pregunta final es: ¿se propone el mismo espacio para las ciencias sociales? En muchos temas que hacen a las problemáticas sociales pareciese que cualquier persona puede opinar, más allá de la credencial académica que posea. Recordemos, a modo de ejemplo temas tales como aquellos relacionados con el género, la violencia social, juventudes, nueva ruralidad, movimientos sociales, vivienda, salud, políticas sociales, pueblos originarios, entre otros. De ninguna manera implica circunscribir el tratamiento de aquellos temas sólo a un círculo de elegidos, por el contrario, si se busca profundizar en las causas de tales problemas, su presentación en la sociedad y sus posibles modos de afrontarlos, es indudable que se recurra a los cientistas sociales que se dedican a estudiar los fenómenos sociales.

Si se comparte que en la aplicación de los denominados derechos de segunda generación hay una estrecha interdependencia con los derechos civiles y políticos (...) los ciudadanos deben disfrutar efectivamente de derechos tales como el derecho al trabajo, a un nivel de vida adecuado, a la salud, la alimentación, la vivienda, la educación, la protección social, el reconocimiento étnico y de identidad cultural, y otros (Hopenhayn, 2005; p.1),

es evidente que las tareas y el resultado de los investigadores de la ciencias sociales pueden aportar mucho para la implementación de políticas públicas en las áreas de gobierno nacional, provincial y municipal. El ejercicio de la ciudadanía no está divorciado de la actividad que realizan los investigadores quienes, junto a su formación científica deben ser ciudadanos responsables y con un profundo sentido ético en sus prácticas. Los cientistas sociales pueden contar con la formación y las herramientas teórico- metodológicas para el estudio de los fenómenos sociales. El desafío no es menor, y el compromiso ciudadano no puede quedar recluido en la academia.

\section{Referencias Bibliográficas}

Aréchiga, H (1994) La ciencia mexicana en el contexto global, en AAVV, México. Ciencia y Tecnología en el umbral del Siglo XXI, México, CONACYT/Porrúa.

Argumedo, A. (1997) Los rasgos de una nueva época histórica. KAIROS-Revista de Temas Sociales. $N^{\circ}$.1. Segundo semestre.

http://www.revistakairos.org/k01-07.htm (pp 89-117) recuperado: 15/05/2012

Bordelois, I. (2003) La palabra amenazada. Buenos Aires. Libros del Zorzal.

Castells, M. (1997) La era de la Información. Vol.1 La sociedad red. España. Alianza Editorial. 
Castro, G. (2000) Cultura política en la cotidianidad de fin de milenio. Kairós, revista de temas sociales. Año 4; $N^{\circ}$ 6. $2^{\circ}$ Semestre.

http://www.revistakairos.org (pp: 1-18) Recuperado: 10/05/2012

(2002) TIC y vida cotidiana. Informática y telecomunicación en la Universidad. El caso de la FICES-UNSL. (Tesis de maestría no publicada). Universidad Nacional de San Luis. Argentina

Conferencia Científica de la VI Cumbre Iberoamericana de Jefes de Estado y de Gobierno, realizada en Santiago de Chile en 1996 Revista Iberoamericana de Educación. Número 12 - Educación y Gobernabilidad Democrática http://www.oei.es/vicumbre.htm. Recuperada: 12/05/2012

Foucault, M. (1985) La hermenéutica del sujeto. Madrid. Ediciones de La Piqueta.

Garcia Canclini, N. (1995) Consumidores y ciudadanos. Conflictos multiculturales de la globalización. México. Editorial Grijalbo.

Giddens, A. (1994) Consecuencias de la modernidad. Madrid. Alianza Universidad.

Guattari, F.; Rolnik, S. (2006) Micropolítica. Cartografías del deseo. Madrid. Edición: Traficantes de Sueños.

Guiñazú, M. C., (2002) Estado y Reforma en las nuevas recomendaciones del Banco Mundial: Conceptos y Teorías. Revista Política y Gestión. Volumen 3. Rosario: Homo Sapiens.

Heller, A. (1985) Historia y vida cotidiana. Una aportación a la sociología socialista. México. Ed. Gijalbo.

(1987) Sociología de la vida cotidiana. Barcelona. Ediciones Península.

(1994) La revolución de la vida cotidiana. Barcelona. Ediciones Península.

Hopenhayn, M. (2005): América Latina desigual y descentrada, Buenos Aires, Norma.

Lemke, J. L (1993) Education, cyberspace and change; en The Arachnet Electronic Journal on Virtual Culture. March 22. Volume 1 Issue 1.

Minayo, M. C. (2009) La artesanía de la investigación cualitativa. Buenos Aires. Lugar Editorial.

Morales Campos, Estela. (2001) La Sociedad de la Información en el siglo XXI y la Biblioteca Universitaria. Revista de la UNAM. Vol.2. No 2.

http://www.revista.unam.mx/vol.2/num2/art1/index.html.

Recuperado: 10/06/2012 
Prigogine, I.; Stengers, I. (1984) La nueva alianza. Metamorfosis de la ciencia. Madrid. Alianza Editorial.

Rovira Mas, J; Rivera, M.; Sader, E.; Gandasegui, H., Marco A. (2009) Edelberto Torres-Rivas: dependencia, marxismo, revolución y democracia. La perspectiva desde la periferia. Crítica y Emancipación, Año 1, Nº 2: (pp. 27-76) primer semestre 2009. Recuperado: 5/06/2012

Sautu, R. (2003) Todo es Teoría: Objetivos y Métodos de Investigación. Buenos Aires. Editorial Lumiére.

Trejo Delarbre, R. (1999) La nueva alfombra mágica. Website:

http://www.etcetera.com.mx/libro/alfombra.htm. Recuperado: 8/06/2012

\section{Sitios institucionales}

Conferencia Científica de la VI Cumbre Iberoamericana de Jefes de Estado y de Gobierno: http:// www.oei.es/vicumbre.htm. Recuperado: 10/06/2012

Ministerio de Ciencia y Tecnología: http://www.mincyt.gov.ar/ministerio/presentacion/index. phpecono Recuperado: 20/08/2012

Agencia Nacional de Promoción Científica y Tecnológica: http://www.agencia.gov.ar/spip. php?article21 Recuperado: 20/08/2012

Programa de Incentivo a Docentes- Investigadores (http://www.me.gov.ar/spu).

Recuperado: 21/08/2012

Comisión Nacional de Evaluación y Acreditación Universitaria (http://www.coneau.edu.ar/coneau/ index.htm(\#).Recuperado: 21/08/2012

Secretaría de Políticas Universitarias del Ministerio de Educación de la Nación: http://www.me.gov. ar/spu/guia tematica/incentivos/incentivo.html Recuperado: 21/08/2012 